\title{
Early Results on the Utilisation of ECG-Imaging During Catheter Ablation Procedures for Prediction of Sites of Earliest Activation During Re-entrant Ventricular Tachycardia
}

\author{
Michele Orini ${ }^{1,2}$, Adam Graham ${ }^{2}$, Mehul Dhinoja ${ }^{2}$, Ross Hunter ${ }^{2}$, Richard J Schilling ${ }^{2}$, Anthony W \\ Chow $^{2}$, Peter Taggart ${ }^{1}$, Pier D Lambiase ${ }^{1,2}$ \\ ${ }^{1}$ University College London, London, United Kingdom \\ 2 Barts Heart Centre, St Bartholomew's Hospital, London, United Kingdom
}

\begin{abstract}
Success rate of ventricular tachycardia (VT) ablation remains sub-optimal. Current technology does not allow fast and accurate delineation of the ablation target. Noninvasive panoramic ECG-imaging (ECGI) offers the possibility of studying the interaction between arrhythmogenic substrate and earliest sites of activation during VT to improve ablation strategies. ECGI mapping (CardioInsight, Medtronic) was performed in 5 patients undergoing VT ablation. Ventricular pacing was delivered from the $R V$ and three indices were measured at each ventricular site to map susceptibility to arrhythmia initiation: Re-entry vulnerability index (RVI), local dispersion of $A T(\triangle A T)$ and local dispersion of repolarization $(\triangle A R I)$. Regions of high susceptibility were defined as those corresponding to the bottom $5 \%$ of RVI and the upper $5 \%$ of $\triangle A T$ and $\triangle A R I$. Morphologically distinct VTs were analyzed to measure the AT sequence and localize the region of earliest epicardial activation $(A T<5 \mathrm{~ms})$. In total, $20 \mathrm{VTs}$ were analyzed $(4.0 \pm 1.2$ per patient). The minimum distance between the region of high vulnerability and the region of earliest AT during VT was $5.6 \pm 8.6 \mathrm{~mm}$ for $R V I$, $6.1 \pm 10.8 \mathrm{~mm}$ for $\triangle A T$ and $12.8 \pm 22.4 \mathrm{~mm}$ for $\Delta A R I$ ( $P>0.13$ for all pair-wise comparison). The vulnerable region presented at least partial overlap with the region of earliest activation during VT in 50\%, 55\% and $50 \%$ of all VTs for $R V I, \triangle A T$ and $\triangle A R I$, respectively. These early data confirm the mechanistic link between markers of arrhythmogenic risk and VT initiation and suggest that ECGI could be potentially used for targeting ablation in non-inducible or hemodynamically non-tolerated VTs.
\end{abstract}

\section{Introduction}

Catheter ablation for ventricular tachycardia (VT) is a medical procedure to treat abnormal fast heart rates and prevent sudden cardiac death in patients with structurally abnormal hearts. Despite much reaserch and technological effort [1], its success rate remains sub-optimal. The purpose of the procedure is to use catheter ablation to modify the substrate for VT initiation and destroy concealed circuits that could support re-entrant VT. The accurate identification of the ablation-target remains challenging [1]. Traditionally, the optimal ablation target is considered to be the site of VT initiation. Current cardiac mapping technology based on sequential contact mapping requires a large number of beats to create voltage or activation/repolarization maps. This is a limitation that hinders the accurate delineation of the arrhythmogenic substrate and increases the duration of the procedure. Electrocardiographic imaging (ECGI) $[2,3]$ is a non-invasive technology that has the potential to overcome this limitation since it computes epicardial extracellular potentials over the left and right ventricles in only one beat, therefore allowing ultra-fast delineation of the arrhythmogenic substrate. In this study, we conducted ECGI studies during catheter ablation for VT and used the data to provide a preliminary evaluation of activation-repolarization metrics for predicting the earliest sites of activation during VT, a proxy of VT initiation site.

\section{Methods}

\subsection{Data Acquisition}

Five patients were studied during catheter ablation for VT at the Barts Heart Centre, London, UK, using the CardioInsight system (Medtronic). Briefly, a vest containing 256 electrodes uniformly covering the patient's torso was used to record body surface potentials (sampling rate 1000 $\mathrm{Hz}$ ) during the entire procedure. Epicardial meshes and electrode locations on the torso were reconstructed in-site from a CT scan performed just before the procedure using commercial software (CardioInsight). Unipolar epicardial electrograms of beats selected by an expert operator dur- 


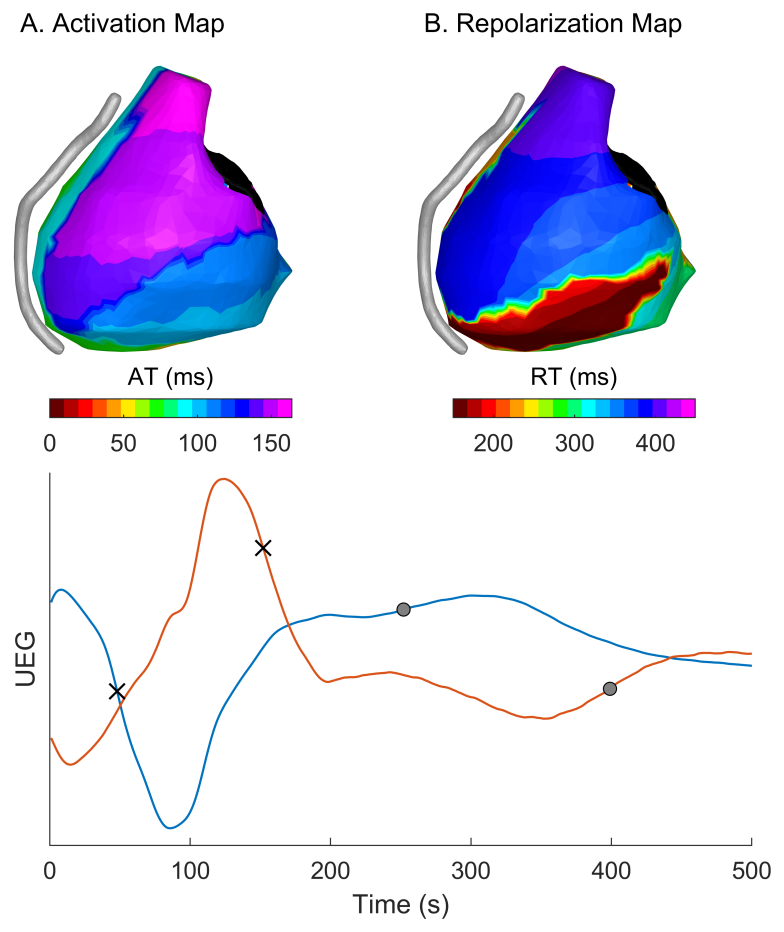

Figure 1. Top: Example of local activation (AT) and repolarization (RT) maps provided by ECG-Imaging (ECGI). The left anterior descending artery is shown in gray. Below: Two unipolar electrograms (UEG) from the same beat, with AT markers shown as a cross $(\times)$ and RT markers shown as a circle $(\bullet)$.

ing the clinical procedure were reconstructed in-site using commercial software (CardioInsight) and exported for offline anlysis. The system estimates the unipolar electrograms by solving the inverse problem of electrocardiography based on previously described algorithms [4]. Typically, more than 1000 electrograms were computed over the entire ventricular epicardium. Exported data included: - Unipolar electrograms recorded during morphologically distinct VTs.

- Unipolar electrograms recorded during a pacing maneuver aimed at inducing conduction slowing/block. Pacing was delivered from the RV apex at a fixed rate of $100 \mathrm{bpm}$ for 5 beats after which an extra beat at a short coupled interval $S_{1} S_{2}=360 \mathrm{~ms}$ was interpolated. The beat after the $S_{1} S_{2}$ pacing interval was analyzed off line.

Data were analyzed with bespoke software as in previous studies [5-8]. After removing the pacing artifacts, local activation time (AT) was measured as the time of the maximum negative downslope during the QRS complex (Figure 1). For local repolarization time (RT) measurements, signals were band-pass filtered between $0.5-25 \mathrm{~Hz}$ and RT was measured as the time of the maximum positive upslope of during the T-wave of the filtered signal. AT and RT were referenced to the earliest AT. The activation-recovery interval, $A R I=R T-A T$, was used as standard surrogate for the local action potential duration.

At each node of the epicardial mesh $i$, neighboring sites $j$ comprised within a $15 \mathrm{~mm}$ radius were identified and the following metrics were computed:

- Re-entry vulnerability index [9]:

$$
R V I_{i}=\min _{j}\left(R T_{i}-A T_{j}\right)
$$

- Local dispersion of activation:

$$
\Delta A T_{i}=\max \left(A T_{j}\right)-\min \left(A T_{j}\right)
$$

- Local dispersion of repolarization:

$$
\Delta A R I_{i}=\max \left(A R I_{j}\right)-\min \left(A R I_{j}\right)
$$

Regions of high susceptibility to re-entry, named as vulnerable regions, were defined as those corresponding to the bottom $5 \%$ of RVI and the upper $5 \%$ of $\triangle A T$ and $\Delta A R I$. Morphologically distinct VTs were analyzed to measure the AT sequence and localize the region of earliest epicardial activation, defined as the region for which $A T \leq 5$ ms. The extension of this region was limited to $5 \%$ of the epicardial nodes. The minimum distance between sites belonging to the vulnerable region and sites belonging to the region of earliest AT during VT, denoted as $D_{m}$, was used to assess the capability of these metrics to predict VT initiation sites.

\section{Results}

In total, $20 \mathrm{VTs}$ were analyzed $(4.0 \pm 1.2$ per patient, range of VT per patient $3-6$ ). An example of AT and RT map provided by ECGI analysis during RV pacing in one patient is shown in Figure 1. Activation and repolarization maps describing electrophysiological dynamics during ventricular pacing were used to measure metrics of vulnerability to re-entry using (1)-(3), while local AT maps during VT were analyzed to identify regions of earliest activation. A representative example is shown in Figure 2-3. Figure 2 shows maps of $R V I, \Delta A T$ and $\triangle A R I$ for the same patient. Interestingly, the vulnerable regions identified by these three indices, represented by white dots, was similar. In Figure 3, panel A shows an RVI map, with red (low RVI values) indicating increased vulnerability to re-entry. The vulnerable region is represented by white dots. Panels B-C show the local AT map of three morphological distinct VTs, with the region of earliest AT shown by black dots. The minimum distance between the two regions, $D_{m}$, is reported above each panel, with $D_{m}=0$ indicating an overlap between them (at least one white dot coincide with a black dot).

Across the 20 VTs considered in this study, the minimum 
A. RVI
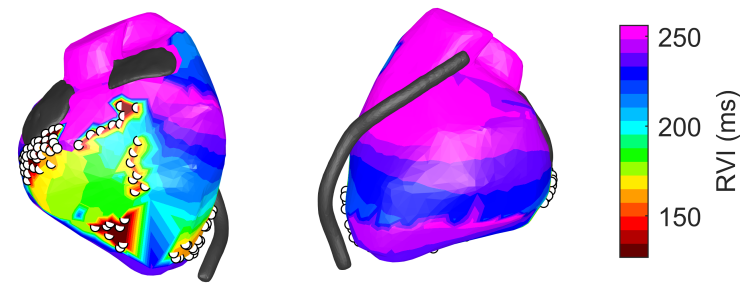

B. $\Delta \mathrm{AT}$
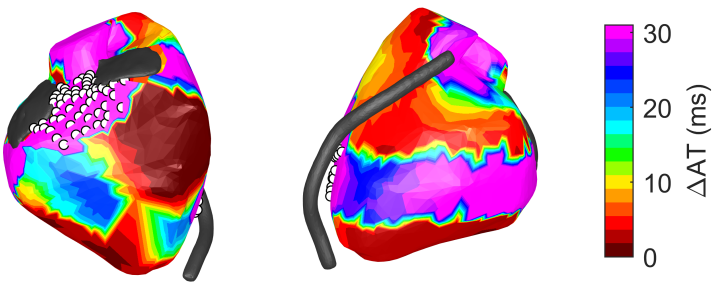

C. $\triangle \mathrm{ARI}$
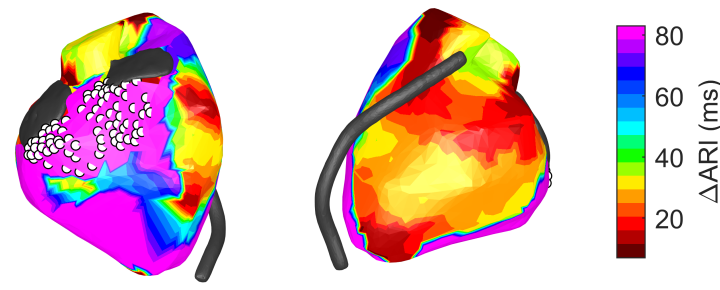

Figure 2. Maps of vulnerability to arrhythmia initiation based on activation-repolarization metrics $R V I$ (top), $\triangle A T$ (middle) and $\triangle A R I$ (bottom), for one patient. Maps are based on data recorded during ventricular pacing at short $S_{1} S_{2}$ pacing interval. Left and right panels show different views of the same heart.

Table 1. Distance $\left(D_{m}\right)$ between the earliest site of activation during VT and the vulnerability region identified during ventricular pacing.

\begin{tabular}{l|cc} 
& $\begin{array}{c}\text { Distance }\left(D_{m}\right) \\
(m \pm S D)\end{array}$ & $\begin{array}{c}d=0 \\
n(\%)\end{array}$ \\
\hline RVI & $5.6 \pm 8.6$ & $10(50 \%)$ \\
$\Delta A T$ & $6.1 \pm 10.8$ & $11(55 \%)$ \\
$\Delta A R I$ & $12.8 \pm 22.4$ & $10(50 \%)$
\end{tabular}

distance between the region of high vulnerability and the region of earliest AT during VT was $5.6 \pm 8.6 \mathrm{~mm}$ for $R V I$, $6.1 \pm 10.8 \mathrm{~mm}$ for $\Delta A T$ and $12.8 \pm 22.4 \mathrm{~mm}$ for $\Delta A R I$ ( $P>0.13$ for all pair-wise comparison). The vulnerable region overlapped with the region of earliest AT during VT (indicating perfect prediction of epicardial breakthrough) in $50 \%, 55 \%$ and $50 \%$ of all VTs for $R V I, \Delta A T$ and $\triangle A R I$, respectively. At least 2 VTs per patients were localized correctly by $R V I$ and $\triangle A T$.
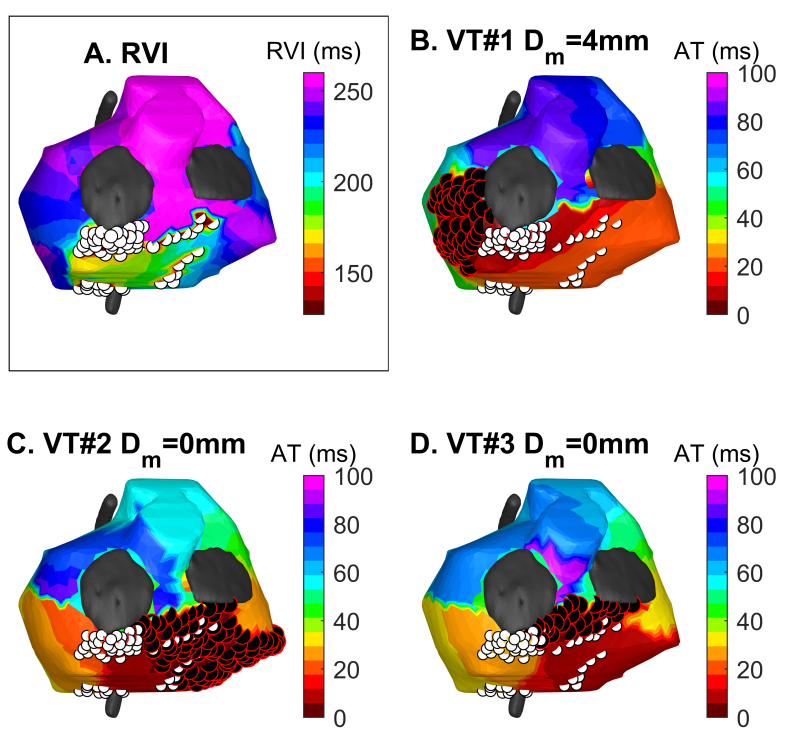

Figure 3. Example of prediction of VT initiation site using RVI in one patient showing 3 morphologically distinct VTs. A: Color map showing RVI, with white dots identifying the vulnerable region $\left(R V I<R V I_{5 \%}\right)$. BD: Local activation time (AT) map during different VTs. Black circles identified the region of earliest activation ( $A T \leq 5 \mathrm{~ms}$ ). The minimum distance between the vulnerable (white dots) and the earliest AT (black dots) regions is equal to $D_{m}$. Note that when white and black dots overlap $D_{m}=0$.

\section{Discussion and Conclusion}

The objective of this study was to provide preliminary results about a novel strategy to predict the cardiac segment most likely to initiate re-entrant VT. We combined ECGI mapping, which provides ultra-fast panoramic mapping of both the RV and LV, with activation-repolarization metrics of tissue vulnerability to arrhythmia. The requirement for the initiation of a re-entrant circuit is that a wavefront of activation traveling along a line of block finds tissue that has already regained excitability, enabling its reactivation. The likelihood that this condition is met increases when the wave-front is slow (long AT) and the wave-back is fast (short RT) [10]. The RVI algorithm [9, 11] provides a point-by-point quantification of this principle, by measuring the difference between RT and AT at pairs of adjacent points throughout the myocardium, with low RVI indicating high vulnerability. RVI has been recently shown to accurately predict the earliest epicardial breakthrough during VT in ARVC and Brugada Syndrome patients [12]. Local dispersion of activation $(\triangle A T)$ is related to heterogeneous conduction and linked to RVI. Local dispersion 
of repolarization $(\triangle A R I)$ is known to promote conduction block, which can facilitate re-entry.

Both RVI and $\triangle A T$ identified regions of high susceptibility to re-entry, with minimum distance of about $6 \mathrm{~mm}$, and at least partial overlap between the vulnerable region and the region of earliest epicardial activation in more than $50 \%$ of VTs. The similarity between results obtained with RVI and $\triangle A T$ indicates that in these patients the vulnerability was likely due to abnormal electrical conduction.

The main limitation of this approach is that ECGI provides only epicardial data. In particular, although the VT exit site may have been in the endocardium, the earliest site of epicardial activation was used to approximate the cardiac segment of VT initiation.

These early data confirm the mechanistic link between markers of arrhythmogenic risk and VT initiation and suggest that ECGI could be potentially used for targeting ablation in non-inducible or hemodynamically non-tolerated VTs. Further studies are needed to assess the value of combining ECGI and activation-repolarization metrics for improving treatment for VT.

\section{References}

[1] Graham AJ, Orini M, Lambiase PD. Limitations and Challenges in Mapping Ventricular Tachycardia: New Technologies and Future Directions. Arrhythmia Electrophysiology Review aug 2017;6(3):118. ISSN 2050-3369.

[2] Rudy Y. Noninvasive electrocardiographic imaging of arrhythmogenic substrates in humans. Circulation Research 2013;112(5):863-874. ISSN 00097330.

[3] Andrews CM, Srinivasan NT, Rosmini S, Bulluck H, Orini M, Jenkins S, Pantazis A, McKenna WJ, Moon JC, Lambiase PD, Rudy Y. Electrical and Structural Substrate of Arrhythmogenic Right Ventricular Cardiomyopathy Determined Using Noninvasive Electrocardiographic Imaging and Late Gadolinium Magnetic Resonance Imaging. Circulation Arrhythmia and Electrophysiology jul 2017; 10(7):e005105. ISSN 19413084.

[4] Ramanathan C, Ghanem RN, Jia P, Ryu K, Rudy Y. Noninvasive electrocardiographic imaging for cardiac electrophysiology and arrhythmia. Nature Medicine apr 2004; 10(4):422-428. ISSN 10788956.

[5] Orini M, Hanson B, Monasterio V, Martínez JP, Hayward
M, Taggart P, Lambiase P. Comparative evaluation of methodologies for T-wave alternans mapping in electrograms. IEEE Trans Biomed Eng 2014;61(2):308-316.

[6] Orini M, Taggart P, Srinivasan N, Hayward M, Lambiase PD. Interactions between activation and repolarization restitution properties in the intact human heart: In-vivo whole-heart data and mathematical description. PLoS ONE 2016;11(9):e0161765.

[7] Orini M, Citi L, Hanson BMB, Taggart P, Lambiase PDP. Characterization of the Causal Interactions Between Depolarization and Repolarization Temporal Changes in Unipolar Electrograms. In Computers in Cardiology, volume 40. IEEE, 2013; 719-722.

[8] Orini M, Taggart P, Lambiase PD. In vivo human sockmapping validation of a simple model that explains unipolar electrogram morphology in relation to conductionrepolarization dynamics. Journal of Cardiovascular Electrophysiology jul 2018;29(7):990-997. ISSN 15408167.

[9] Child N, Bishop MJ, Hanson B, Coronel R, Opthof T, Boukens BJ, Walton RD, Efimov IR, Bostock J, Hill Y, Rinaldi CA, Razavi R, Gill J, Taggart P. An activationrepolarization time metric to predict localized regions of high susceptibility to reentry. Heart Rhythm 2015; 12(7):1644-53.

[10] Coronel R, Wilms-Schopman FJ, Opthof T, Janse MJ. Dispersion of repolarization and arrhythmogenesis. Heart Rhythm 2009;6(4):537-543.

[11] Orini M, Taggart P, Hayward M, Lambiase P. Optimization of the global re-entry vulnerability index to minimise cycle length dependency and prediction of ventricular arrhythmias during human epicardial sock mapping. In Computing in Cardiology, volume 44. ISSN 2325887X, sep 2017;

[12] Martin CA, Orini M, Srinivasan NT, Bhar-Amato J, Honarbakhsh S, Chow AW, Lowe MD, Ben-Simon R, Elliott PM, Taggart P, Lambiase PD. Assessment of a conductionrepolarisation metric to predict Arrhythmogenesis in right ventricular disorders. International Journal of Cardiology may 2018;ISSN 18741754.

Address for correspondence:

Name: Michele Orini

Full postal address: University College London, Gower Street, London, UK.

E-mail address: m.orini@ucl.ac.uk 